www.nature.com/pj

\title{
Molecular dynamics simulations for microscopic behavior of water molecules in the vicinity of zwitterionic self-assembled monolayers
}

\author{
Ryo Nagumo ${ }^{1}$, Tatsunori Ito ${ }^{2}$, Kazuki Akamatsu ${ }^{3}$, Ryuji Miura ${ }^{2}$, Ai Suzuki ${ }^{1}$, Hideyuki Tsuboi ${ }^{1}$, \\ Nozomu Hatakeyama ${ }^{2}$, Hiromitsu Takaba ${ }^{2}$ and Akira Miyamoto ${ }^{1,2}$
}

Polymer Journal (2012) 44, 1149-1153; doi:10.1038/pj.2012.72; published online 9 May 2012

Keywords: betaine; biocompatibility; bound water; free water; molecular dynamics; polyzwitterion; protein adsorption

\section{INTRODUCTION}

Zwitterionic biomaterials, such as phosphobetaine polymers, ${ }^{1}$ have been widely used because of their excellent biocompatibility, which results from suppression of nonspecific protein adsorption and cell adhesion. $^{2-5}$ To understand fundamentally how the biocompatible materials can prevent protein adsorption at the molecular level, significant efforts have been made by experimental approaches. Ishihara et al. ${ }^{1}$ investigated the hydration of zwitterionic 2-methacryloyloxyethyl phosphorylcholine (MPC) polymers, indicating that MPC can hold water molecules strongly. Feng et al. ${ }^{2}$ suggested that the water layer above the MPC surface does not change the native conformation of adsorbed proteins. Kitano et al. ${ }^{3}$ concluded that a 1-carboxy- $N, N$-dimethyl- $N-\left(2^{\prime}\right.$-methacryloyloxyethyl) methanaminium inner salt $(\mathrm{CMB})$ monomer does not change the hydrated structure of water molecules. These experimental reports have suggested that the structure of water in the vicinity of zwitterionic biomaterials definitely influences suppression of protein adsorption.

Molecular dynamics (MD) simulations have also been a powerful approach for evaluating the molecular behaviors in various fields, ${ }^{6,7}$ including protein adsorption phenomena on various material surfaces. $^{8-11}$ Chen et $a l .{ }^{8}$ reported the strong resistance of zwitterionic phosphorylcholine (PC) self-assembled monolayers (SAMs) to protein adsorption. He et al. ${ }^{9}$ also investigated the antifouling properties of PC SAMs by MD simulations. They suggested that their strong resistance to biofouling results from their capacity for hydration via electrostatic interactions. Water molecules in the vicinity of the SAM surface probably act to produce a strong repulsive force on the approaching protein, because of a tightly bound water layer. They also suggested that these tightly bound layers are due to the ionic solvation nature of zwitterionic head groups, by comparing with the data obtained for a nonionic oligo(ethylene glycol) (OEG) SAM surface. The PC and OEG SAMs are found to be quite different in the antifouling mechanisms. Water molecules in the hydration layer near the PC SAM surface stay longer than those on the OEG SAM surface. This means that water molecules are more tightly bound on the PC SAM surface. Furthermore, the water molecules near the PC SAM surface is much more randomly oriented, which is close to bulk water. They conclude that the PC SAM surface binds water by ionic electrostatic interactions more strongly than the hydrophilic and neutral OEG SAM surface, where water molecules are more loosely bound via hydrogen bonding.

These previous studies ${ }^{8,9}$ are significant for the elucidation of mechanisms on the development of zwitterionic PC materials, but at the same time, a new interest arises in microscopic behaviors of water molecules in the vicinity of various types of material, including zwitterionic and nonionic ones. In this work, MD simulations are conducted to investigate the dynamics and structural properties of explicit water molecules near three types of zwitterionic SAM surface, PC, carboxybetaine (CB) and sulfobetaine (SB), as well as poly(vinyl alcohol) (PVA) and graphite surfaces. Residence times and orientational distributions are analyzed for each type of surface to gain a microscopic insight into thermodynamic mechanisms on the suppression of protein adsorption on zwitterionic biomaterials.

\section{MATERIALS AND METHODS}

In this study, PC, CB and SB SAMs consist of thiol linear monomers, whose chemical structural formulae are shown in Figure 1a. We modeled these SAM surfaces whose areas are $61 \times 53 \AA^{2}$, where $8 \times 8$ linear monomers were located in parallel. The height of all these models was set to $60 \AA$. Using the cell of the same size as the SAMs, we also modeled PVA and graphite surfaces. In our PVA model, seven linear chain monomers, consisting of eleven vinyl alcohol

${ }^{1}$ New Industry Creation Hatchery Center, Tohoku University, Sendai, Japan; ²Department of Chemical Engineering, Graduate School of Engineering, Tohoku University, Sendai, Japan and ${ }^{3}$ Department of Environmental and Energy Chemistry, Faculty of Engineering, Kogakuin University, Tokyo, Japan 


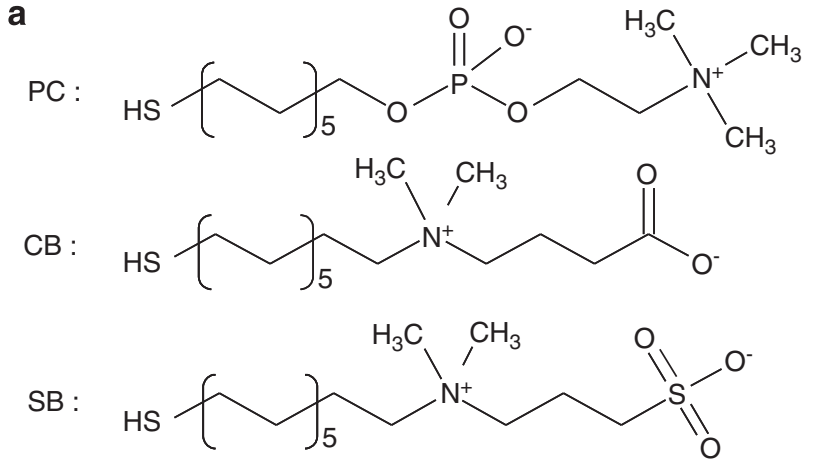

b

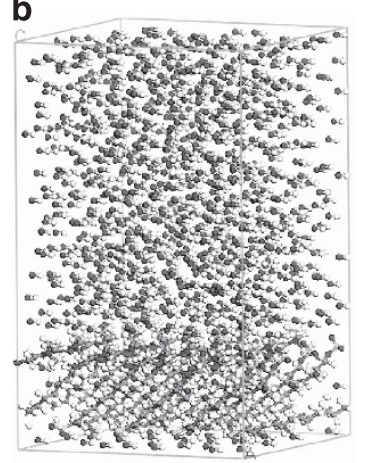

C

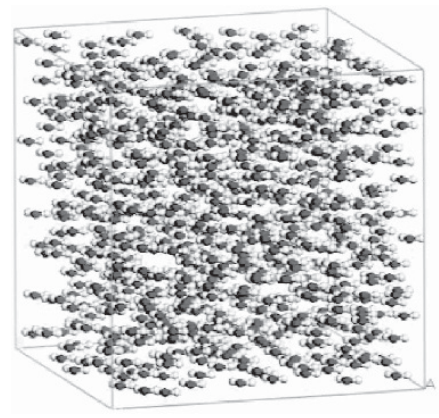

Figure 1 (a) Chemical structural formulas of PC SAM, CB SAM and SB SAM repeat units. (b) Simulation cell of PC SAM. (c) Simulation cell of bulk water. A full color version of this figure is available at Polymer Journal online.

repeat units, were parallel located, as the density of PVA becomes ca. $1.26 \mathrm{~g} \mathrm{~cm}^{-3}$ (http://www.polymerprocessing.com/polymers/PVOH.html). It is noted that our PVA model is not amorphous and regularly oriented, as well as the other SAM surfaces, although the ideally structured model can be used for reference. For modeling the graphite surface, two graphite layers, where each layer is composed of 346 carbon atoms, were replicated. Using grand canonical Monte Carlo (GCMC) simulations, 1174, 1143, 1126, 1159 and 1283 numbers of water molecules were inserted into PC SAM, CB SAM, SB SAM, PVA and graphite cells, respectively, where the densities of the liquid phases are set to ca. $1 \mathrm{~g} \mathrm{~cm}^{-3}$. Figure $1 \mathrm{~b}$ shows the PC SAM models used in this study. For comparison, we also considered a $30 \times 30 \times 30 \AA^{3}$ cubic cell, where no material surfaces are contained but 835 water molecules are inserted to set the density of nearly $1 \mathrm{~g} \mathrm{~cm}^{-3}$, as shown in Figure 1c. Here the cell is called a bulk water model.

The newly updated all-atom AMBER force field ${ }^{12,13}$ was adopted to describe the interactions for the materials and water molecules (http://ambermd.org/\#ff). Nonbonded van der Waals interactions were described with a 12-6 LennardJones potential, which was truncated at $15.0 \AA$. A periodic boundary condition was applied in all three directions. In all our MD simulations, an NVT ensemble (number of atoms, volume and total energy in the simulation box are kept constant) was applied and the temperature was set at $300 \mathrm{~K}$. The time steps for each MD step were $0.1 \mathrm{fs}$. For the SAM models, the sulfur atoms within thiol groups of each SAM chain were fixed during MD simulations. For the PVA and graphite model, all the carbon atoms were fixed. After the minimization by 50000 steps of MD calculations after the GCMC insertion, more 50000 steps of MD were performed for each model to analyze the structural and dynamic properties of water molecules.

In estimating these properties, we considered four hydration layers equally spaced from the water/material interface, whose thicknesses are $4 \AA$, as illustrated in Figure 2a. Here the hydration layer which contacts the surface is denoted by the 'first' layer, while the next ones are denoted by the second, third and fourth layers. The residence time of water molecules is an important dynamic property, because it directly correlates the affinity between a material
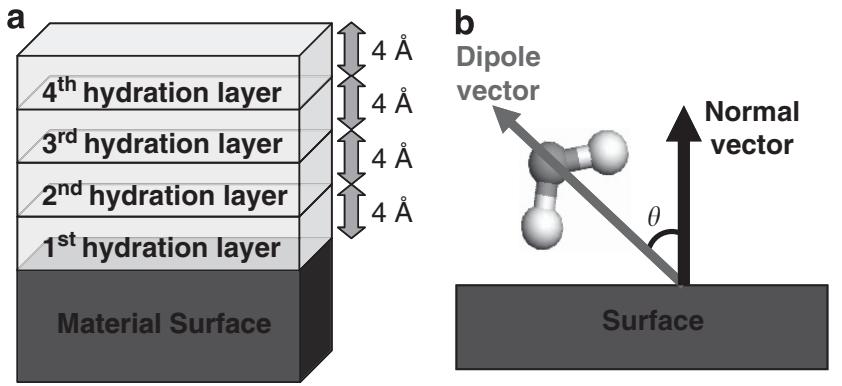

Figure 2 (a) Schematic of equally spaced hydration layers in the vicinity of material surfaces. (b) Schematic of the angle $\theta$ between the dipole vector of a water molecule and the surface normal vector. A full color version of this figure is available at Polymer Journal online.

surface and water. This property is determined by the proportion of water that stays in each layer with an elapsed time. It is calculated from the survival time correlation function, ${ }^{14} C(t)$, given by

$$
C(t)=\frac{1}{N} \sum_{i=1}^{N} \frac{\left\langle P_{i}(0) P_{i}(t)\right\rangle}{\left\langle P_{i}(0)\right\rangle^{2}}
$$

where $P_{i}(t)$ is a binary function whose value is 1 if the $i$ th water molecule remains in a hydration layer for the time $t$ without going out of the layer. $P_{i}(t)$ is estimated for all water molecules initially contained in that layer. $N$ denotes the number of these water molecules. The brackets $\langle\ldots\rangle$ are the ensemble average. Considering the definition of $C(t)$, its slower decrease means that a material surface tends to bind water molecules more strongly. Besides the residence time, the orientations of the water molecules in the vicinity of a surface were evaluated by the angle $\theta$ between its dipole vector and the surface normal vector, ${ }^{9}$ as illustrated in Figure $2 \mathrm{~b}$. In the case of a bulk water model, molecules are randomly oriented, because all dipole angles are equally possible, resulting in the flat orientation distribution.

\section{RESULTS}

Figure 3 shows the correlation functions over the elapsed time for each hydration layers in the vicinity of the PC SAM, CB SAM, SB SAM, PVA and graphite surfaces. The correlation function for the bulk water was also shown. In the first hydration layer, the correlation function for PC SAM decreases to nearly 0.2 after 5 ps. This is more drastic reduction, as compared with the previous report for PC SAM, ${ }^{9}$ where it is only reduced to about 0.8 at the equivalent time. This remarkable difference results from the fact that a protein molecule is located at the separation distance of $5 \AA$ above a PC SAM surface in the previous report. The protein can be definitely a steric barrier for water molecules to move freely near the surface, leading to much higher values of correlation functions. Therefore, Figure 3 shows the trends of the correlation functions can change depending on surrounding environments.

As shown in Figure 3a, in the first hydration layer, the PVA surface seems to bind water molecules more strongly than the other surfaces, suggesting that a hydration layer is formed in the vicinity of nonionic and hydrophilic types of surface. For the PC SAM surface, the water molecules stay nearly as long as those on the PVA until ca. 3 ps, but they tend to separate more than those on PVA, after 3 ps. CB and SB SAMs also bind water molecules, and the values of the correlation functions are larger than those of bulk water, particularly after the run time of 1 ps. It seems strange that water molecules on the PVA surface stay longer than those on zwitterionic SAMs, because electrostatic interactions on zwitterionic SAMs should be stronger than hydrogen bonding, and plausible reasons are discussed below. Around the graphite layers, the water molecules can move more freely than bulk water molecules, indicating that the exchange of the positions of the water molecules is quite frequent on hydrophobic interfaces. 

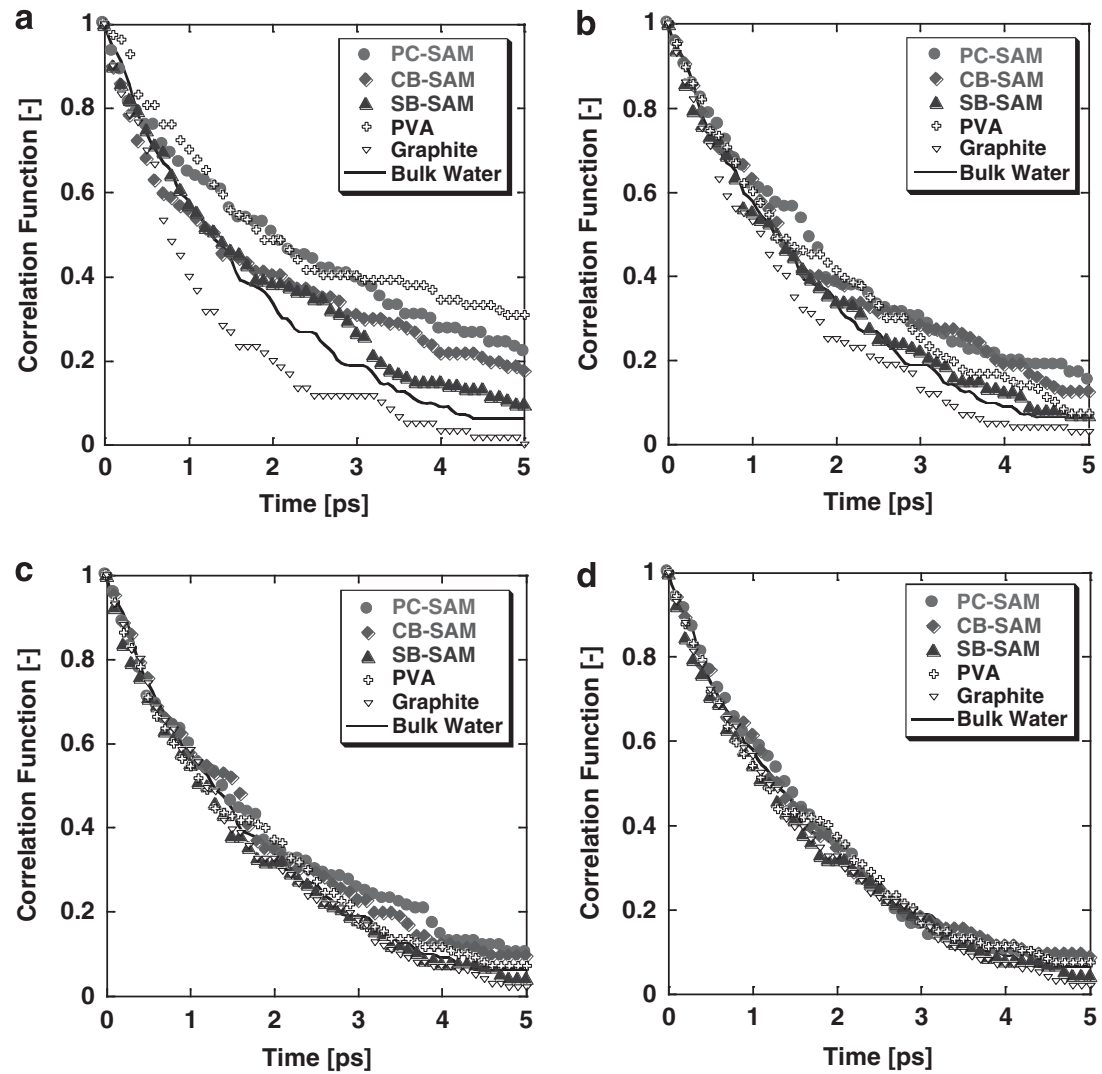

Figure 3 Elapsed time dependence of correlation functions for each of the four hydration layers near the PC SAM, CB SAM, SB SAM, PVA and graphite surfaces in (a) the first, (b) second, (c) third and (d) the fourth layer. For reference, the function for the bulk water was also added. A full color version of this figure is available at Polymer Journal online.

On the other hand, as shown in Figure 3b, the correlation function in the second hydration layer for the PVA surface drastically decreases, as compared with that for the first hydration layer. The values of the functions for PC and CB SAMs become larger than those for PVA, particularly after the run time of 3 ps. This reverse indicates that hydration networks near the PVA surface, probably via hydrogen bonding, drastically diminish in the second hydration layer. In the zwitterionic SAMs, the absolute values of the functions in the second layer do not decrease very much, as compared with those in the first layer. This suggests that the second hydration layer, which is mainly formed by stronger ionic electrostatic interactions than hydrogen bonding, ${ }^{9}$ has a key role in antifouling properties of betaine materials. As for the correlation functions for the third and fourth hydration layers, differences between the types of material have gradually diminished and become similar to those for bulk water.

Figures $4 \mathrm{a}$ and $\mathrm{b}$ show orientational distributions of the water molecules for the first and second hydration layers, respectively. The distributions are clearly different between PVA, graphite and zwitterionic surfaces in the first layer, whereas the difference nearly disappears in the second layer. As for the PVA surfaces, water molecules are highly oriented and the maximum value of the probability appears around at $50-60^{\circ}$. Considering the $\mathrm{H}-\mathrm{O}-\mathrm{H}$ angle of a water molecule is generally about $104.5^{\circ}$, the water molecules near PVA tend to form highly structured networks via hydrogen bonding, as illustrated in Figure 4c. In this ideal scheme, oxygen and hydrogen atoms of water molecules lie alternatively on a straight line. In the second layer, on the other hand, the network clearly diminishes and the water molecules become randomly oriented, as is case with bulk water. These results are reinforced by the data of the residence times in Figure 3, which clarifies the reverse of the correlation functions for the PVA and the PC SAM and CB SAM surfaces between the first and second hydration layers. Considering that ionic electrostatic interactions hardly acts on the water molecules near the nonionic PVA surfaces, protein molecules can easily invade the second hydration layer and can probably contact the first layer. The water molecules near the graphite surface are also oriented and the maximum value of the distribution probability is around at $90^{\circ}$, as schematically illustrated in Figure $4 \mathrm{~d}$. In this case, van der Waals interactions between graphite surfaces and a water molecule certainly become the most dominant among the possible orientations, leading to higher repulsion between them. This repulsion promotes the positional exchange between water molecules near the interface. Therefore, in protein molecules approaching the graphite surface, the adsorption would be remarkably induced.

For the three types of betaine SAM surface, the water molecules are randomly oriented in both the first and second hydration layers. The result for PC SAM is in good agreement with that in the previous study. ${ }^{9}$ The PC SAM surface certainly forms a strong hydration layer via ionic electrostatic interaction, suggesting that the randomly oriented water molecules has a significant role in preventing proteins from approaching the surface.

\section{DISCUSSION}

In this study, the structural and dynamical properties of the water molecules in the vicinity of graphite, PVA and three types of zwitterionic SAMs were investigated, by analyzing the residence times 

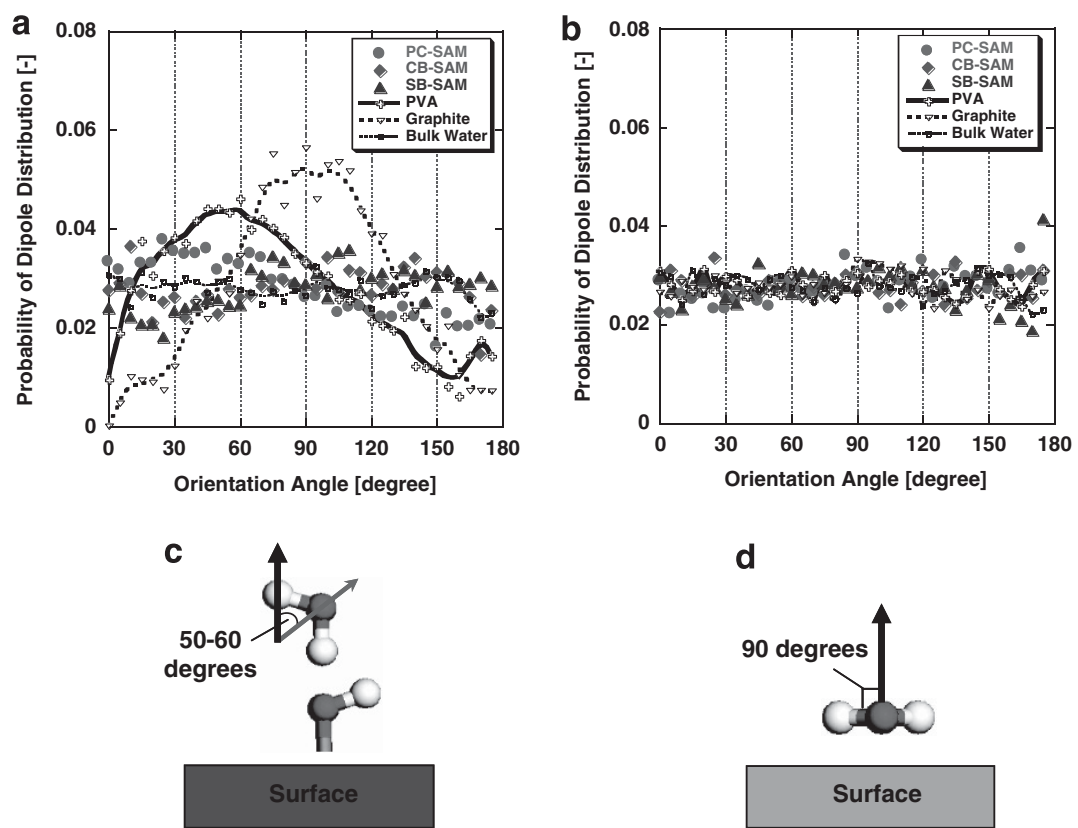

Figure 4 Distribution of the dipole angles for the water molecules near the PC SAM, CB SAM, SB SAM, PVA and graphite surfaces in (a) the first and (b) the second layer. For reference, that for the bulk water was also added. (c) Schematic of a highly oriented hydration network, where the dipole angle is around $50-60^{\circ}$, in the vicinity of the PVA surface. (d) The dipole angle is $90^{\circ}$. This is possible in the case of the water molecules near the graphite interface. A full color version of this figure is available at Polymer Journal online.

and the orientational distributions. Taking into account only Figure $3 \mathrm{a}$, it is possible that the PVA surface can suppress the protein adsorption most effectively among all the types of surface in this study. However, considering the reverse in the trend of the correlation functions between Figures $3 \mathrm{a}$ and $\mathrm{b}$, and the orientational change for the PVA surface between Figures $4 \mathrm{a}$ and $\mathrm{b}$, the structured hydration networks via hydrogen bonding are limited within the first layer on the PVA/water interface. Therefore, proteins can certainly border the first layer. Such a situation is difficult to image for the vicinity of the zwitterionic SAM surfaces, considering the results of the residential times in Figures $3 \mathrm{a}$ and $\mathrm{b}$. Moreover, the water molecules near the PVA surface are highly oriented, as seen in Figure 4a. When a protein approaches the PVA surface, the total entropy in the system increases, because the orientations of the surrounding water molecules become more random, resulting in a decrease in the total free energy in the system. As a result, the promotion of protein adsorption is energetically favorable on the PVA surfaces. This is exactly the same as the graphite surface, because the water molecules are also highly oriented in its vicinity. Around the zwitterionic SAMs, on the other hand, the water molecules are randomly oriented, as shown in Figures $4 \mathrm{a}$ and b. It means that even though the proteins approach the zwitteionic SAM surface, the total entropy hardly changes, and that the total free energy in the system probably remains almost unchanged. The explanation is derived from Figures $3 \mathrm{a}$ and $\mathrm{b}$ and Figures $4 \mathrm{a}$ and $\mathrm{b}$ that the thicker hydration layer near the zwitterionic SAM surfaces, formed via stronger ionic interactions through the two hydration layers on the interface, is certainly more robust, as compared with the thinner layer formed via weaker hydrogen bonding observed for the PVA surface. These discussions are consistent with the previous experimental reports ${ }^{1-4}$ that the zwitterionic SAM surfaces can suppress nonspecific protein adsorption. It should be noted that our results were obtained from the simulations on the nanosecond time scale, and that the longer time scales need to be considered to estimate the precise amount of overall effective water molecules.

As a next step to achieve a theoretical prediction of antifouling properties for various materials, it is essential to calculate the free energies in the system by considering several approaches, such as high-temperature configuration-space exploration and replica exchange method. ${ }^{15-17}$

\section{ACKNOWLEDGEMENTS}

This work has been supported by the research project, 'Application of integrated intelligent satellite system to construct regional water resources utilization system,' sponsored by Core Research for Evolutional Science and Technology of the Japan Science and Technology Agency (JST).

1 Ishihara, K., Nomura, H., Mihara, T., Kurita, K., Iwasaki, Y. \& Nakabayashi, N. Why do phospholipid polymers reduce protein adsorption? J. Biomed. Mater. Res. 39, 323-330 (1998)

2 Feng, W., Zhu, S., Ishihara, K. \& Brash, J. L. Adsorption of fibrinogen and lysozyme on silicon grafted with poly(2-methacryloyloxyethyl phosphorylcholine) via surfaceinitiated atom transfer radical polymerization. Langmuir 21, 5980-5987 (2005).

3 Kitano, H., Tada, S., Mori, T., Takaha, K., Gemmei-Ide, M., Tanaka, M., Fukuda, M. \& Yokoyama, Y. Correlation between the structure of water in the vicinity of carboxybetaine polymers and their blood-compatibility. Langmuir 21, 11932-11940 (2005).

4 Chen, S. G., Chen, S. J., Jiang, S., Mo, Y. M., Luo, J. X., Tang, J. N. \& Ge, Z. C. Study of zwitterionic sulfopropylbetaine containing reactive siloxanes for application in antibacterial materials. Colloid Surf. B: Biointerfaces 85, 323-329 (2011).

5 Akamatsu, K., Mitsumori, K., Han, F. \& Nakao, S. Fouling-free membranes obtained by facile surface modification of commercially available membranes using the dynamic forming method. Ind. Eng. Chem. Res. 50, 12281-12284 (2011).

6 Nagumo, R., Takaba, H. \& Nakao, S. Application of free energy calculations at an ultrahigh temperature for estimation of molecular diffusivities and permeabilities in zeolite nanopores at an ambient temperature. J. Phys. Chem. B 113, 13313-13321 (2009).

7 Onodera, T., Morita, Y., Nagumo, R., Miura, R., Suzuki, A., Tsuboi, H., Hatakeyama, N., Endou, A., Takaba, H., Dassenoy, F., Minfray, C., Joly-Pottuz, L., Kubo, M., Martin, J. M. \& Miyamoto, A. A computational chemistry study on friction of h-MoS 2 . Part II. Friction anisotropy. J. Phys. Chem. B 48, 15382-15838 (2010). 
8 Chen, S. F., Zheng, J., Li, L. Y. \& Jiang, S. Y. Strong resistance of phosphorylcholine self-assembled monolayers to protein adsorption: insights into nonfouling properties of zwitterionic materials. J. Am. Chem. Soc. 127, 14473-14478 (2005).

9 He, Y., Hower, J., Chen, S. F., Bernards, M. T., Chang, Y. \& Jiang, S. Y. Molecular simulation studies of protein interactions with zwitterionic phosphorylcholine self-assembled monolayers in the presence of water. Langmuir 24, 10358 (2008).

10 Raffaini, G. \& Ganazzoli, F. Protein adsorption on a hydrophobic surface: a molecular dynamics study of lysozyme on graphite. Langmuir 26, 5679-5689 (2010).

11 Raut, V. P., Agashe, M. A., Stuart, S. J. \& Latour, R. A. Molecular dynamics simulations of peptide-surface interactions. Langmuir 21, 1629-1639 (2005).

12 Pearlman, D. A., Case, D. A., Caldwell, J. W., Ross, W. S., Cheatham, T. E., Debolt, S., Ferguson, D., Seibel, G. \& Kollman, P. AMBER. A package of computer-programs for applying molecular mechanics, normal-mode analysis, molecular-dynamics and free-energy calculations to simulate the structural and energetic properties of molecules. Comput. Phys. Comm 91, 1-41 (1995).

13 Case, D. A Cheatham, T. E., Darden, T., Gohlke, H., Luo, R., Merz, K. M., Onufriev, A. Simmerling, C., Wang, B. \& Woods, R. J. The Amber biomolecular simulation programs. J. Comput. Chem. 26, 1668-1688 (2005).

14 Dastidar, S. G. \& Mukhopadhyay, C. Structure, dynamics, and energetics of water at the surface of a small globular protein: a molecular dynamics simulation. Phys. Rev. E 68, 021921 (2003).

15 Sugita, Y. \& Okamoto, Y. Replica-exchange molecular dynamics method for protein folding. Chem. Phys. Lett. 314, 141-151 (1999).

16 Nagumo, R., Takaba, H. \& Nakao, S. I. High-accuracy estimation of 'slow' molecular diffusion rates in zeolite nanopores, based on free energy calculations at an ultrahigh temperature. J. Phys. Chem. C 112, 2805-2811 (2008).

17 Schüring, A., Auerbach, S. M. \& Fritzsche, S. A simple method for sampling partition function ratios. Chem. Phys. Lett. 450, 164-169 (2007). 